\title{
74 TUMOR MICROENVIRONMENT BASED ON PD-L1 AND CD8 T-CELL INFILTRATION CORRELATED WITH THE RESPONSE OF MSS MCRC PATIENTS TREATED VACTOSERTIB IN COMBINATION WITH PEMBROLIZUMAB
}

\begin{abstract}
${ }^{1}$ Tae Won Kim*, ${ }^{2}$ Keun-Wook Lee, ${ }^{3}$ Joong Bae Ahn, ${ }^{4}$ Young Suk Park, ${ }^{5}$ Chan-Young Ock, ${ }^{5}$ Hyejoo Park, ${ }^{5}$ Jiyeon Ryu, ${ }^{5}$ Bitna Oh, ${ }^{5}$ Bo-Kyoung Kim, ${ }^{5}$ Sunjin Hwang, ${ }^{5}$ Ki Baik Hahm, ${ }^{5}$ Seong-Jin Kim. ${ }^{1}$ Asan Medical Center, University of Ulsan College of Medicine, Seoul, Korea, Republic of; ${ }^{2}$ Seoul National University Bundang Hospital, Seoul National University College of Medicine, Seoul, Korea, Republic of; ${ }^{3}$ Yonsei Cancer Center, Yonsei University College of Medicine, Seoul, Korea, Republic of; ${ }^{4}$ Samsung Medical Center, Sungkyunkwan University School of Medicine, Seoul, Korea, Republic of; ${ }^{5}$ MedPacto, Inc., Seoul, Korea, Republic of
\end{abstract}

Background The expression of PD-L1 and tumor-infiltrating $\mathrm{CD} 8 \mathrm{~T}$ cells were reported to have a decisive effect on the immunotherapy response (PMID: 26819449). Microsatellite stable (MSS) metastatic colorectal cancer (mCRC) shows a limited clinical benefit to immunotherapy alone known to be having an 'immune-cold' microenvironment. To understand the basis of the clinical responses to anti-PD- 1 and TGF- $\beta$ inhibitor combination therapy in MSS mCRC, we conducted a comprehensive analysis of survival outcome, whole transcriptome sequencing (WTS) data, and multiplex immunohistochemistry $(\mathrm{mIHC})$ data from a combination treatment of vactosertib and pembrolizumab.

Methods Clinical outcomes were evaluated by RECIST v1.1 and iRECIST. Tumor tissue biopsy samples for WTS and mIHC were obtained in screening and cycle 2 post-treatment time point. CD274(PD-L1) and CD8A expression cut-off were calculated as a median value in the merged data set of TCGA Pan-cancer and MP-VAC-204 study. Having over median value defined as a high group and under median value as a low group. Tumor immune status by a combination of gene expression (high or low) was classified into four subtypes (1: CD274 high, CD8A high; 2: CD274 low, CD8A low; 3: CD274 high, CD8A low; 4: CD274 low, CD8A high). Tumor tissue slides stained with PD-L1, CD8, and granzyme B (GZB) in tumor nest and stroma.

Results Among 43 patients whose WTS data are available, thus included in the analysis, 9 patients were responders (7 PRs and 2 iPRs). Subtype 2 showed a major proportion in the MP-VAC-204 CRC patients (1: 14\%, 2: 58\%, 3: 12\%, 4: $16 \%)$. Responders were observed in subtype 2 and 4 (24\% and 14\%, RECIST). The CD8A expression and median overall survival (mOS) showed a significantly positive correlation (** $\mathrm{P}=0.0028)$ and there was no significance in the correlation of CD274 and mOS. mOS was significantly longer in high expression of CD8A patients ( $\mathrm{P}=0.0083$, Not reached vs 9.9 months, hazard ratio 8.812 [95\% CI 3.19-24.31]). After the combination treatment, CD8 and GZB positive T cells were increased significantly in both tumor nest and stroma.

Conclusions Our study suggests a high level of CD8 T cells, even in the case of low PD-L1 expression, is significantly correlated with the improved clinical outcomes in MSS mCRC patients treated with vactosertib and pembrolizumab. The increases in CD8 $\mathrm{T}$ cells both in tumor nest and stroma after the combined inhibition of PD- 1 and TGF- $\beta$ pathway may contribute to the survival benefit. Further clinical investigations are warranted. (Clinical trial information: NCT03724851)

http://dx.doi.org/10.1136/jitc-2021-SITC2021.074 